\title{
Mechanical Characterization of Human Brain Tissue and Soft Dynamic Gels Exhibiting Electromechanical Neuro-Mimicry
}

Anthony Tabet, Stefan Mommer, Julian Vigil, Clement Hallou, Harry Bulstrode, Oren A. Scherman*

Synthetic hydrogels are an important class of materials in tissue engineering, drug delivery, and other biomedical fields. Their mechanical and electrical properties can be tuned to match those of biological tissues. In this work, we report on hydrogels that exhibit both mechanical and electrical biomimicry. The presented dual networks consist of supramolecular networks formed from 2:1 homoternary complexes of imidazolium-based guest molecules in cucubit[8]uril and covalent networks of oligoethylene glycol(di)methacrylate. We also investigate the viscoelastic properties of human brain tissues. The mechanical properties of the dual network gels are benchmarked against the human tissue, and we find that they both are neuro-mimetic and exhibit cytocompatiblity in a neural stem cell model.

The development of robust and facile synthetic materials for applications into bioelectronics, ${ }^{[1]}$ optogenetics, ${ }^{[2]} 3 \mathrm{D}$ cell culture, ${ }^{[3,4]}$ and drug delivery ${ }^{[5,6]}$ is an active field of research. In particular, well defined synthetic materials that possess biophysical, mechanical, and electrical mimicry of tissue are of wide interest for applications such as machinebrain interfaces. ${ }^{[7-9]}$ Hydrogels are a prominent class of materials that combine many of the aforementioned properties. ${ }^{[3,10-14]}$ Their stiffness is tunable and their chemical functionality allows for versatile modifications enabling cell adhesion and protein sequestration. ${ }^{[4,15]}$ Furthermore, they provide an ideal environment for cells facilitating nutrient transport, due to their high water uptake. Nonetheless, limited attention has been given to properties such as electrical resistivity or ion conductivity in biomimetic hydrogel systems.

The electrical properties of hydrogels can play a crucial role in applications inside and outside of biomedicine. ${ }^{[1]}$ The majority of conductive hydrogels are based on electrically conductive polymers where the delocalized $\pi$-electrons move freely along the conjugated backbone. Prominent examples include polythiophene (PT), polypyrrole (PPy),

\footnotetext{
A. Tabet, Dr. S. Mommer, J. Vigil, Prof. O.A. Scherman

Melville Laboratory for Polymer Synthesis,

Department of Chemistry, University of Cambridge

Lensfield Road, Cambridge, CB2 1EW, UK

A. Tabet, C. Hallou, Dr. H. Bulstrode

Department of Paediatrics,

Addenbrooke's Hospital, University of Cambridge

Hills Road, Cambridge CB2 0QQ, UK

E-mail: hb252@cam.ac.uk, oas23@cam.ac.uk
}

poly(3,4-ethylene dioxythiophene) (PEDOT) and polyaniline (PANi). ${ }^{[16-19]}$ Other types of conductive materials include polyelectrolytes and poly(ionic liquids), which have charged monomers furnishing the final (co)polymer with pendant ionic side groups, such as carboxylic acid, quarternary ammonium, and imidazolium salts. ${ }^{[20]}$ These conductive materials are of great interest regarding the development of soft, biomimetic electronic materials. Our group recently reported on the advantages of using soft hydrogels as alternatives to stiff wafers for adjuvant local chemotherapy against glioblastoma. ${ }^{[21]}$ The advantages of a soft material interfacing with the brain include (i) greater epitaxial engagement, and (ii) mitigated long-term neurological side effects that occur from the stiffness mismatch with tissue.

We recently reported on the electromechanical properties and supramolecular structure of stiff and conductive hydrogels made from statistical copolymers of acrylamide (Aam) and 1-benzyl-3-vinylimidazolium (VIm). ${ }^{[22]}$ The previously synthesized hydrogels were not cytocompatible but possessed excellent electrical properties by virtue of the ionic guest. In separate studies, the mechanical properties of soft, dual networks based on incorporation of VIm moieties into the polymeric chains were explored. ${ }^{[23,24]}$ VIm molecules form 2:1 homoternary complexes with cucubit[8]uril (CB[8]; Fig. S1), which facilitates the percolation of a supramolecular network. ${ }^{[22,25]}$ Here we build on our previous work and report VIm/oligoethylene glycol (OEG) based materials that exhibited cytocompatibility with a mouse neural stem cell model. In creating these synthetic materials, we were inspired by Owens et al., who argue that biomimicry of tissue must include electromechanical properties. ${ }^{[26,27]}$ Here, we report the first data on the oscillatory rheological properties of the human brain and gliomas at $37^{\circ} \mathrm{C}$, and we benchmark these properties against our gels.

These VIm/OEG/CB[8] polymer networks were synthesized via free radical polymerization, which enabled control of monomer composition and in situ cross-linking of the polymer solution by use of both supramolecular and covalent cross-linking motifs. Irgacure 2595 (2-Hydroxy4-(2-hydroxyethoxy)-2-methylpropiophenone) was used as water-soluble photoinitiator. Due to both strong electrostatic repulsion and steric hindrance of neighboring VIm units, the ionic monomer has a low reactivity ratio with itself $\left(k_{1,1} \sim 0\right.$; Fig. S2), and consequently cannot be homopolymerized. In the polymerization process, a total of $5 \mathrm{~mol} \%$ VIm guest was randomly incorporated in the polymer backbone. Next, 0.5 

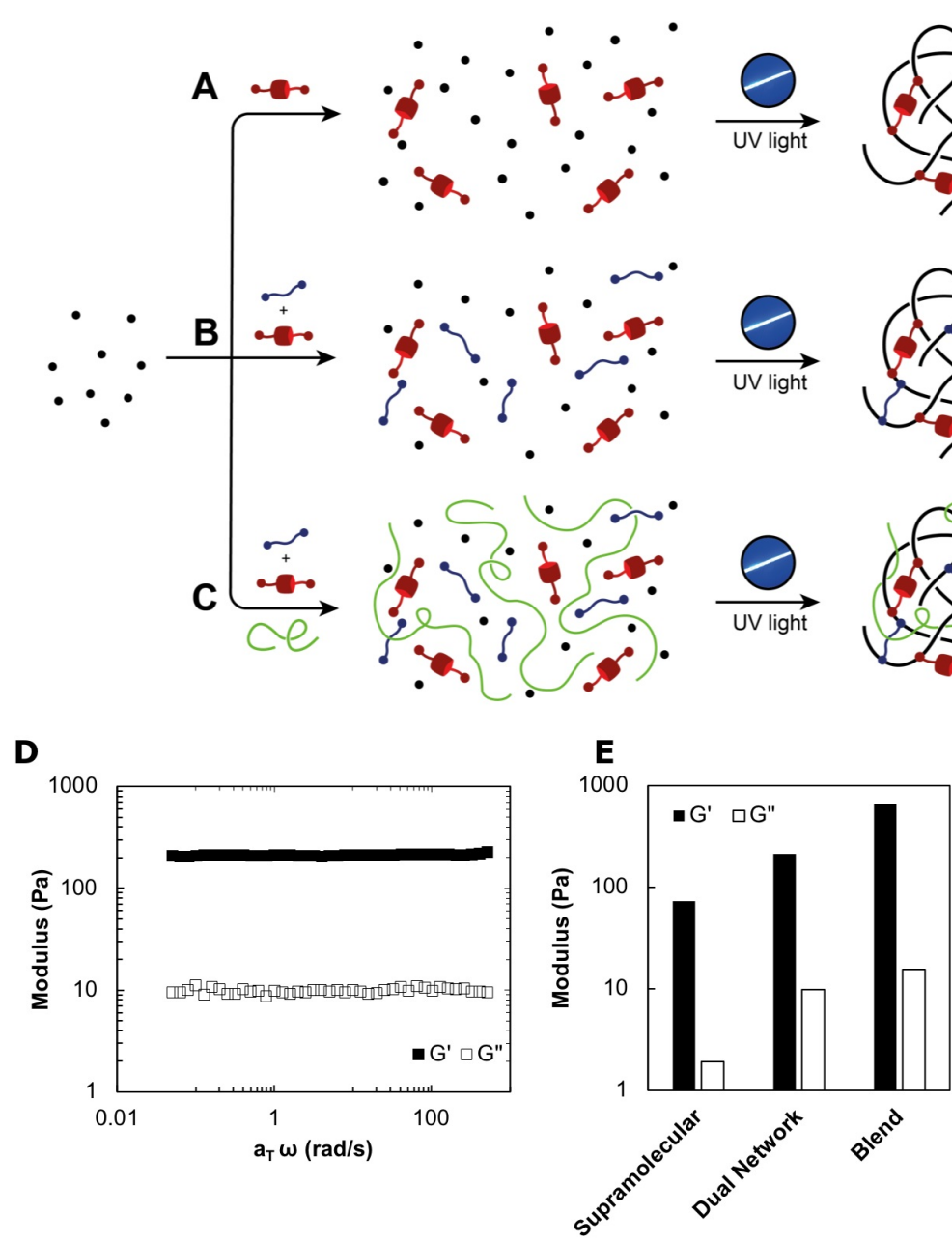

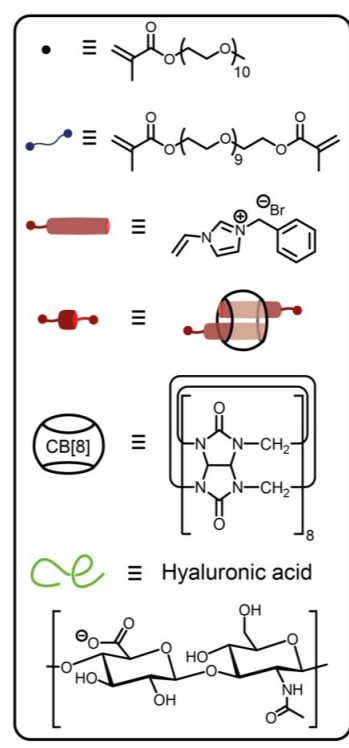

$\mathbf{F}$

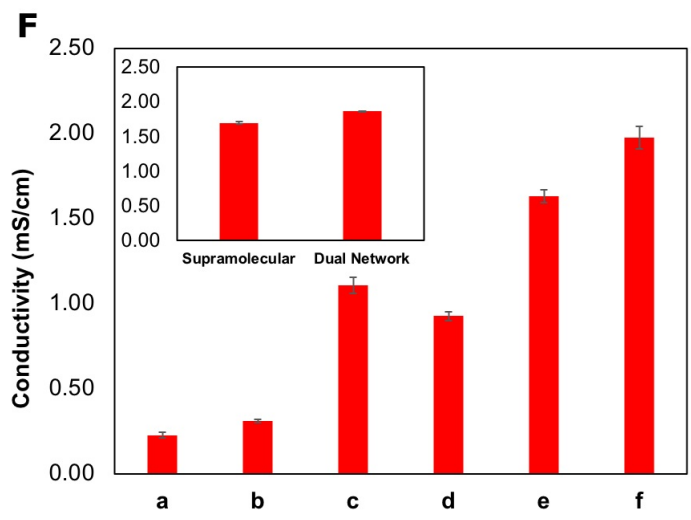

Figure 1 Illustration of the synthetic route used to form (A) supramolecular gels, (B) dual networks and (C) dual networks blended with hyaluronic acid. Characterization of VIm/OEG based hydrogels. (D) Oscillatory frequency sweep of dual network at $37^{\circ} \mathrm{C}$. (E) Storage and loss moduli of supramolecular, dual, and blended gels at $1 \mathrm{rad} / \mathrm{s}$ and $37^{\circ} \mathrm{C}$. (F) Conductivity of hydrogels from PEIS measurements. $\boldsymbol{a}$ Milli-Q water, $\boldsymbol{b}$ covalent OEG network with no ionic species, $\boldsymbol{c}$ VIm/OEG system with no CB[8], $\boldsymbol{d}$ OEG system with HA, $\boldsymbol{e}$ VIm/OEG system with $\mathrm{CB}[8], f$ VIm/OEG system with HA. $n=3$; mean \pm standard deviation.

equivalents (relative to VIm) of $\mathrm{CB}[8]$ were added, and the resulting 2:1 homoternary complexes led to transient physical cross-links between polymer chains (Fig 1A). In the case of dual network gels, OEG-dimethacrylate (OEGDMa) was added as additional covalent cross-linker (Fig 1B). To explore the mechanisms of conductivity in these systems, a negatively-charged linear polysaccharide, hyaluronic acid (HA), was blended in at a 1:1 molar ratio to VIm and the electrical properties of this system was compared to the dual network gels. Hyaluronic acid is the only supramolecular glycosaminoglycan, and its non-covalent interactions with a charged matrix is a property of interest. ${ }^{[12]} \mathrm{HA}$ allowed further elucidation of the electrical properties in the systems described here.

The mechanical properties of these gels were first evaluated with oscillatory rheology (Fig. 1D-E, S3). To elucidate the role of the covalent cross-linker OEGDMa, the storage and loss moduli of the supramolecular gels were compared to the those of the dual network. The supramolecular gel had a storage modulus $\mathrm{G}^{\prime}=72 \mathrm{~Pa}$ and a loss modulus $\mathrm{G}^{\prime \prime}=2 \mathrm{~Pa}$. Upon introduction of 0.05 molar equivalents of the OEGDMa crosslinker, $\mathrm{G}^{\prime}$ increased to $212 \mathrm{~Pa}$ and $\mathrm{G}^{\prime \prime}$ to $10 \mathrm{~Pa}$, all at $1 \mathrm{rad} / \mathrm{s}$ and $37{ }^{\circ} \mathrm{C}$. If 0.05 molar equivalents of HA $\left(M_{n}=1.5-1.8 \mathrm{MDa}\right)$ were blended into the system, it stiffened again to yield a heightened $\mathrm{G}^{\prime}$ and $\mathrm{G}^{\prime \prime}$ of $650 \mathrm{~Pa}$ and $20 \mathrm{~Pa}$, respectively.

The hydrogels' electrical properties were characterized via potentiostatic electrochemical impedance spectroscopy (PEIS). Materials were placed between two planar copper electrodes and a sinusoidal AC potential was applied about the open circuit potential of the cell at different frequencies. The current-frequency response was measured and modelled after the impedance in a characteristic electrical circuit (Fig. S4). A modified Randles circuit was chosen to model the frequency response of the gels to evaluate the solution resistance and calculate conductivity (Fig. 1F, S5). Milli$\mathrm{Q}$ water and a covalent OEG network without any charged 
groups showed negligible conductivity. When equimolar repeat units of VIm or HA are added to these systems, the conductivity similarly increased to $1.11 \pm 0.05 \mathrm{mS} / \mathrm{cm}$ and $0.93 \pm 0.3 \mathrm{mS} / \mathrm{cm}$, respectively, due to diffusion of the ion. When $\mathrm{CB}[8]$ is introduced into the VIm system, the conductivity increased to $1.63 \pm 0.04 \mathrm{mS} / \mathrm{cm}$, possibly due to the addition of charge transfer between imidazolium units. ${ }^{[22,23]}$ When HA is blended into the VIm/CB[8] system, the conductivity non-linearly increases to $2.00 \pm 0.07 \mathrm{mS} / \mathrm{cm}$. This non-additive increase in the conductivity suggests HA, a negatively-charged polymer, may interact with the positively charged backbone of the dual network and retard some $\pi-\pi$ interactions between imidazolium units. Alternatively, or perhaps in tandem, the diffusion of ions may be mitigated due to increased resistance to mass transfer. These impedance and rheology data are consistent with electrostatic interactions between charged species in the system. Furthermore, the conductivity of these dual network hydrogels were comparable to the conductivity of the brain. ${ }^{[28,29]}$
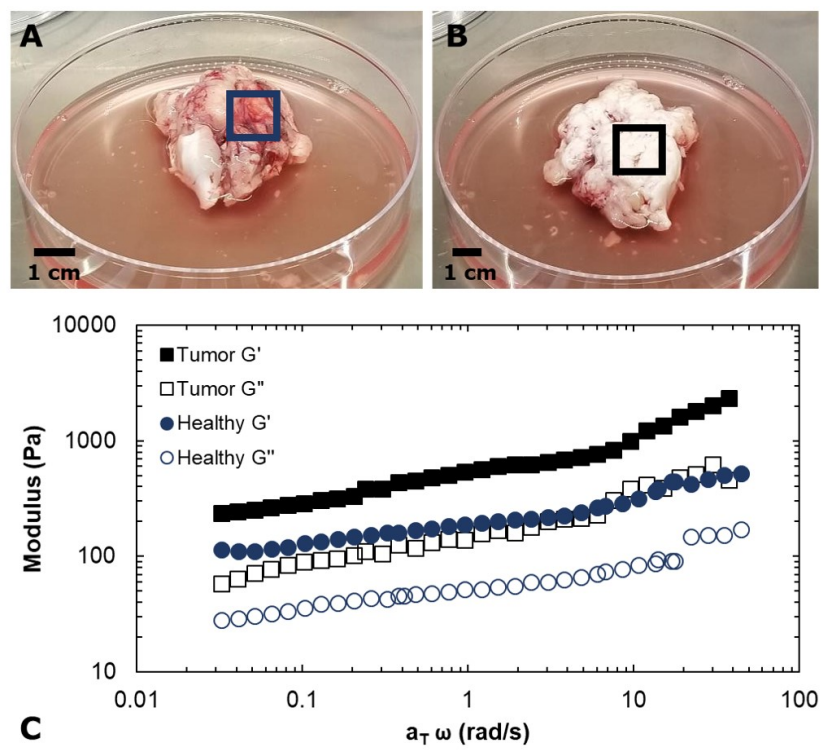

Figure 2 Oscillatory rheological analysis of resected human brain tissues. (A-B) images from resected tumor indicated region of rheological analysis (peripheral or white matter). (C) Oscillatory rheological measurements of human brain and tumor at $37^{\circ} \mathrm{C}$.

To evaluate the mechanical similarity between these systems and human brain tissue, oscillatory rheological measurements were performed on resected human brain tissue (Fig. 2). This body of data is the first to explore such mechanical properties on healthy human brain and human glioblastoma (GB) tissue. An example of such a tumor is shown in Fig. 2A-B. The peripheral, highly vascularized resection consisting of predominantly non-GB (healthy) tissue was compared to central white-matter (tumor) tissue at $37{ }^{\circ} \mathrm{C}$. The storage modulus of the healthy tissue at $1 \mathrm{rad} / \mathrm{s}$ was $189 \mathrm{~Pa}$, compared to that of the tumor tissue, which was $536 \mathrm{~Pa}$ at the same frequency. It is well known that GB tissue will be stiffer than healthy or peripheral tissue, as was recently reported at room temperature. ${ }^{[21]}$ These data show that the hydrogels can be tuned to span the range of healthy tissue to tumor stiffnesses, which may be useful for the development of healthy or glioma organoid models.

The shear-resistance of healthy human brain tissue and dual network gels were then examined (Fig. S6). The samples were exposed to cycles of low and high strains and frequencies. Tissue dehydrated quickly (ESI), so the same frequency was not chosen in order to provide appreciable shear for $1 \mathrm{~min}$. We previously published on the shear-resistance that dual network systems based on VIm/CB[8] chemistry possess. ${ }^{[22-24]}$ These data on our dual network systems and tissue are in agreement with our previous observations and suggest that unlike purely covalent hydrogels, the addition of supramolecular interactions such as host-guest or electrostatic interactions enable more shear-resistive systems.

These bioelectronic materials were evaluated for cytotocompatibility with adult mouse neural stem cells. Imidazolium-based materials have received much attention recently, ${ }^{[22,23]}$ and this is the first data exploring the cellular behavior of such ionic supramolecular and covalent materials. Neural stem cells were used both as a tractable model system and because they closely reflect the transcription program underlying glioblastoma stem cell growth. Supramolecular and dual network gels were added at different concentrations in cell culture media. The stem cells were then incubated with this modified media and brightfield images were taken every $3 \mathrm{~h}$. Quantitative kinetic growth data was taken and plotted in Fig. S8 against positive and negative controls. When cells became $80-90 \%$ confluent, they were fixed and nuclei stained for sox 2 and cas 3 , respectively (Fig. S9-S10). Adult mouse neural stem cells divide every $24 \mathrm{~h}$, which allows for a rapid readout. ${ }^{[30]}$ Nonbiocompatible materials may trigger apoptosis and result in the stagnation or reduction of confluence over time as a result of cell death, or it may lower the rate of cellular growth. At $2000 \mu \mathrm{g} / \mathrm{mL}$ concentration, no change in the rate of proliferation or terminal confluency was observed in either the supramolecular or dual network systems. Examination of cellular morphology via brightfield images also qualitatively suggested the materials were cytocompatible with this model system.

Immunohistochemistry was then used to quantify the expression of neural stem cell transcription factor sox 2 and apoptotic marker cas3. At terminal confluence, the neural stem cells were fixed and stained with primary and secondary antibodies for confocal imaging. The cells expressed high amounts of sox 2 , and minimal $(<1 \%)$ expressed cas 3 . Such data suggest these gels, which are made from FDAapproved building blocks, showed no adverse biological effect against a mouse neural stem cell model. Of course, in vivo evaluation is required over several months to elucidate the biocompatiblity and what, if any, adverse affects may arise from long-term implantation of such an electronic gel. Nonetheless, these confluence and immunohistochemistry data are promising pre-clinical results of a new class of 
A

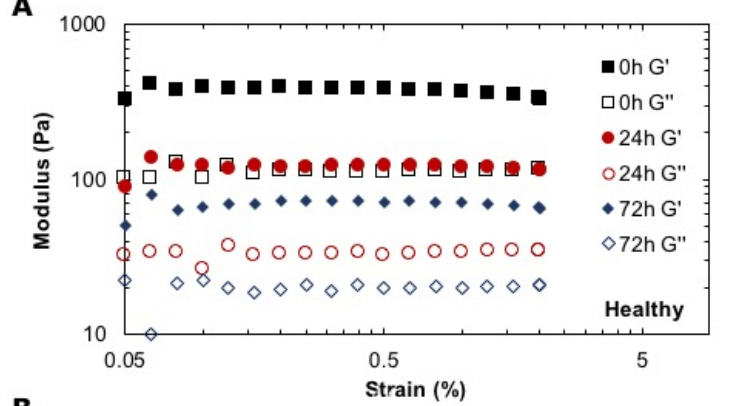

B
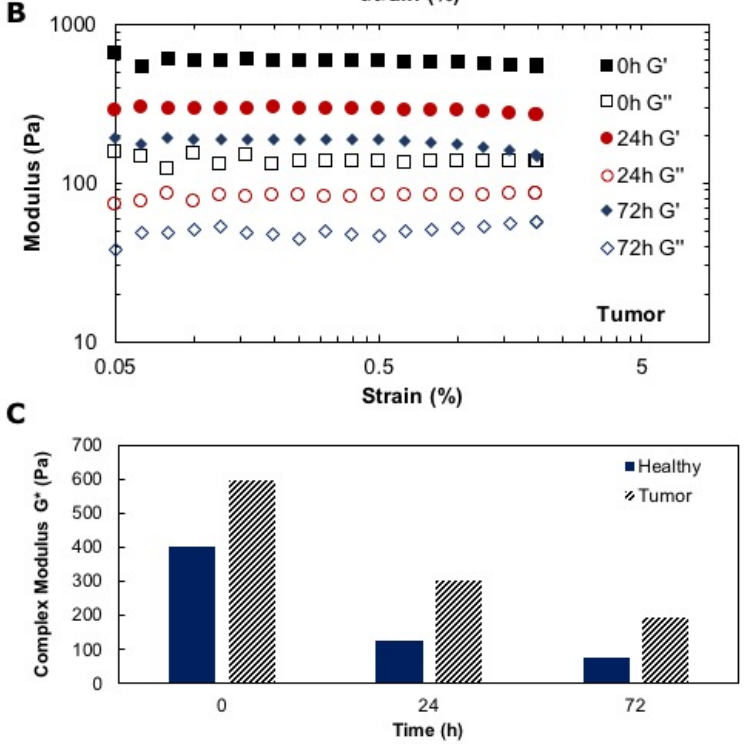

Figure 3 Time resolved rheological analysis of human brain and tumor tissue. (A-B) Time resolved strain sweeps of brain tissues showing dramatic decreases across $24 \mathrm{~h}$ and $72 \mathrm{~h}$ at $20^{\circ} \mathrm{C}$. (C) Complex moduli of tissues at $1 \mathrm{rad} / \mathrm{s}$ over time at $20^{\circ} \mathrm{C}$.

soft electromechanically neuro-mimetic hydrogels.

Unlike covalent or dual network synthetic hydrogel matrices, which are generally stable over time barring any embedded degradability, the human brain (Fig. S9) rapidly changes after resection or traumatic injury. The brain has a high cell volume fraction $(\sim 80 \%) .{ }^{[31]}$ To understand the time-resolved mechanical properties of tissue after resection, we measured the stiffness of healthy and tumor tissue at 3 time points and found major reductions in stiffness in both healthy and tumor samples (Fig. 3). After $24 \mathrm{~h}$, the tumor tissue storage modulus reduced 2-fold (590 Pa to $294 \mathrm{~Pa}$ ), while the healthy tissue reduced more than 3-fold (386 Pa to $124 \mathrm{~Pa}$ ). Further reductions were observed at $72 \mathrm{~h}$. This rapid decrease in stiffness is consistent with rapid necrosis of human brain. Such data provides insight into the structureproperty relationship in human brain tissue and the role that cells play in the overall mechanical properties of central nervous tissue.

In summary, VIm/OEG/CB[8] supramolecular and dual network bioelectronic hydrogels were synthesized and their mechanical, electrical, and biological properties were characterized. The mechanical properties of human brain tissue were also reported, and these data were used to benchmark the neuro-mimicry of VIm/OEG/CB[8] gels. Dual network gels showed similar electromechanical properties to the human brain. These experiments are the first instance of electrically and biologically characterizing soft networks formed via $\mathrm{VIm} / \mathrm{OEG} / \mathrm{CB}[8]$ chemistry. Such materials may find suitable future applications within soft electronics.

\section{Acknowledgements}

A.T. and J.V. thank The Winston Churchill Foundation of the United States. S.M. thanks the Newton International Fellowship. The authors thank Prof. David Rowitch, Dr. Vijay Rana, and Jarrod Shilts (Cambridge) for useful scientific discussions.

\section{Ethical Approval}

The analysis of resected tissue and brain MRI acquisition was approved by the Health Research Authority (England) and the Cambridge University Hospitals NHS Foundation Trust (IRAS ref: 238683). Written, informed consent from patients was obtained.

\section{References}

[1] T. Someya, Z. Bao, G. Malliaras, Nature 2016, 540, 379-385.

[2] P. Anikeeva, Nat. Biotechnol. 2016, 34, 43-44.

[3] K. Y. Lee, D. J. Mooney, Chem. Rev. 2001, 101, 1869-1879.

[4] J. J. Rice, M. M. Martino, L. De Laporte, F. Tortelli, P. S. Briquez, J. A. Hubbell, Adv. Healthcare Mater. 2013, 2, 57-71.

[5] A. Tabet, C. Wang, Adv. Healthcare Mater. 2018, 1800908.

[6] A. Tabet, M. Jensen, C. C. Parkins, P. G. Patil, C. Watts, O. A. Scherman, Adv. Healthcare Mater. 2018, 1801391.

[7] A. Canales, X. Jia, U. P. Froriep, R. A. Koppes, C. M. Tringides, J. Selvidge, C. Lu, C. Hou, L. Wei, Y. Fink, P. Anikeeva, Nat. Biotechnol. 2015, 33, 277-284.

[8] S. P. Lacour, G. Courtine, J. Guck, Nat. Rev. Mater. 2016, 1, 1-14.

[9] J. Rivnay, H. Wang, L. Fenno, K. Deisseroth, G. Malliaras, Sci. Adv. 2017, 3, e1601649.

[10] N. A. Peppas, J. Z. Hilt, A. Khademhosseini, R. Langer, Adv. Mater. 2006, 18, 1345-1360.

[11] N. Annabi, A. Tamayol, J. A. Uquillas, M. Akbari, L. E. Bertassoni, C. Cha, G. Camci-Unal, M. R. Dokmeci, N. A. Peppas, A. Khademhosseini, Adv. Mater. 2014, 26, 85-124.

[12] A. Tabet, K. Sokolowski, J. Shilts, M. Kamp, N. Warner, D. Hoogland, O. A. Scherman, F1000Research 2018.

[13] A. Tabet, R. A. Forster, C. C. Parkins, G. Wu, O. A. Scherman, Polym. Chem. 2019, 10, 467-472.

[14] C. Soo Yun Tan, G. Agmon, J. Liu, D. Hoogland, E.-R. Janeček, E. A. Appel, O. A. Scherman, Polym. Chem. 2017, 8, 5336.

[15] C. M. Madl, S. C. Heilshorn, Adv. Funct. Mater. 2018, 28, 1706046.

[16] T. H. Qazi, R. Rai, A. R. Boccaccini, Biomaterials 2014, 35, 90689086.

[17] A. Guiseppi-Elie, Biomaterials 2010, 31, 2701-2716. 
[18] Y. Zhao, B. Liu, L. Pan, G. Yu, Energy Environ. Sci. 2013, 6, 28562870 .

[19] T. Nezakati, A. Seifalian, A. Tan, A. M. Seifalian, Chem. Rev. 2018, 118, 6766-6843.

[20] J.-L. Barrat, F. Joanny in Adv. Chem. Phys. Wiley-Blackwell, 2007, pp. 1-66.

[21] M. J. Rowland, C. C. Parkins, J. H. McAbee, A. K. Kolb, R. Hein, X. Jun Loh, C. Watts, O. A. Scherman, Biomaterials 2018, 179, 199_ 208 .

[22] J Liu, C Soo Yun, Z Yu, Y Lan, C Abell, O. A. Scherman, Adv. Mater. 2017, 1605325.

[23] J Liu, C Soo Yun, Z Yu, Y Lan, C Abell, O. A. Scherman, Adv. Mater. 2017, 1604951.

[24] J. Liu, C. Soo Yun Tan, Y. Lan, O. A. Scherman, J. Polym. Sci. A1 2017, 55, 3105-3109.
[25] S. J. Barrow, S. Kasera, M. J. Rowland, J. Del Barrio, O. A. Scherman, Chem. Rev. 2015, 115, 12320-12406.

[26] S. Inal, A. Hama, M. Ferro, C. Pitsalidis, J. Oziat, D. Iandolo, A. Pappa, M. Hadida, M. Huerta, D. Marchat, P. Mailley, R. Owens, Adv. Biosyst. 2017, 1, 1700052.

[27] R. Marc, H. Adel, H. Miriam, R. Jonathan, L. Pierre, R. Owens, Adv. Mater. 2014, 26, 7083-7090.

[28] S. Gabriel, R. Lau, C. Gabriel, Phys. Med. Biol. 1996, 41, 2251.

[29] L. Manola, B. H. Roelofsen, J. Holsheimer, E. Marani, J. Geelen, Med. Biol. Eng. Comput. 2005, 43, 335-343.

[30] D. Danovi, A. A. Folarin, B. Baranowski, S. M. Pollard, Methods in Enzymology 2012, 506, 311-29.

[31] C. Nicholson, E. Sykova, Trends Neurosci. 1998, 21, 207-215. 